\title{
F0X01, a Potential Therapeutic Target, Regulates Autophagic Flux, Oxidative Stress, Mitochondrial Dysfunction, and Apoptosis in Human Cholangiocarcinoma QBC939 Cells
}

\author{
Wei He $\mathrm{H}^{\mathrm{a}, \mathrm{e}}$ Aiqing Zhang ${ }^{\mathrm{b}}$ Lei $\mathrm{Q}^{\mathrm{a}}$ Chen $\mathrm{Na}^{\mathrm{c}}$ Rui Jiang ${ }^{\mathrm{d}}$ Zhining Fan ${ }^{\mathrm{C}}$ \\ Jianping Chen ${ }^{a}$
}

\begin{abstract}
aDepartment of Gastroenterology, the Third Affiliated Hospital of Soochow University, Changzhou, bDepartment of Pediatric Nephrology, the Second Affiliated Hospital of Nanjing Medical University, Nanjing, 'Department of Pediatrics, Yanan Hospital Affiliated to Kunming Medicical University, Kunming, dSpecila wards, the Third Affiliated Hospital of Soochow University, Changzhou, eDigestive Endoscopy Center, The First Affiliated Hospital with Nanjing Medical University, and Jiangsu Province Hospital , Nanjing, PR China
\end{abstract}

\section{Key Words}

Foxo1 - Cholangiocarcinoma Autophagy - Oxidative stress $\cdot$ Mitochondrial dysfunction

\begin{abstract}
Background/Aims: Autophagy is an evolutionarily conserved catabolic mechanism to maintain energy homeostasis and to remove damaged cellular components, which plays an important role in the survival of various cells. Inhibiting autophagy is often applied as a new strategy to halt the growth of cancer cells. Methods: The effect of FOXO1 gene on cellular function and apoptosis and its underlying mechanisms were investigated in cultured QBC939 cells by the methylthiazoletetrazolium (MTT) assay, western blot, DCFDA mitochondrial membrane potential, and ATP content measurement. FOXO1 siRNA was applied to down-regulate FOXO1 expression in QBC939 cells. Results: Here we reported that FOXO1, acetylation of FOXO1 (AcFOXO1) and the following interaction between Ac-FOXO1 and Atg7 regulated the basal and serum starvation (SS)-induced autophagy as evidenced by light chain 3 (LC3) accumulation and p62 degration. Either treatment with FOXO1 siRNA or resveratrol, a sirt1 agonist, inhibited autophagic flux, resulting in oxidative stress, mitochondrial dysfunction (MtD) and apoptosis in QBC939 cells, which were attenuated by enhancing autophagy with rapamycin. On the contrary, inhibiting autophagic flux with 3-MA worsened all these effects in QBC939 cells. Conclusions: Taken together, our study for the first time identified FOXO1 as a potential therapeutic target to cure against human cholangiocarcinoma via regulation of autophagy, oxidative stress and MtD.

W. He and A. Zhang contributed equally to this work.

Jianping Chen

and Zhining Fan




\section{Introduction}

The incidence of cholangiocarcinoma is growing over the past decades [1, 2], and relative resistance of the tumors to chemotherapy leading to poor outcome is a thorny problem in patients with cholangiocarcinoma $[3,4]$. Molecular insights into pathophysiology of cholangiocarcinoma cells and novel therapeutic strategies are urgent for the improved clinical management of patients with cholangiocarcinoma.

Macroautophagy (hereafter referred to as autophagy) is an evolutionarily conserved catabolic mechanism to maintain energy homeostasis and to remove damaged cellular components [5, 6]. FOXO1 is a member of the forkhead box O (FOXO) family, which a series of recent studies have demonstrated plays an important role in several intracellular functions, including autophagy [7], cell cycle [8], apoptosis [9], and tumor suppression [10]. Various experiments pointed out that post-translational modifications regulated FOXO1 transcriptional activities and modulated FOXO1 protein stability, DNA binding activity, protein-protein interactions, and subcellular localization, all of which are involved in regulating autophagic flux $[7,11]$.

Oxidative stress, characterized by increased ROS production, plays a key role in the regulation of cells survival [12]. Many drugs exert their negative effects on cancer cells are through increased the production of ROS $[13,14]$. Mitochondrial dysfunction (MtD) represents a malfunction in biochemical processes, characterized by MMP collapse and decreased ATP production, also have an essential role in mediation of apoptosis $[15,16]$. Impaired autophagic flux could result in oxidative stress and MtD, both of which represents a malfunction in biochemical processes $[17,18]$.

Therefore, the main aim of this study was to elucidate whether FOXO1 existed in autophagy regulation and whether impairing autophagic flux could lead to oxidative stress and MtD in QBC939 cells. Finally, we explored the possibility that FOXO1 served as the therapeutic target to cure human cholangiocarcinoma.

\section{Materials and Methods}

\section{Materials}

The established QBC939 was obtained from Cell Bank of Wuhan University (Wuhan, China). Resveratrol, Chloroquine (CQ), and rapamycin (RP) were from Sigma Chemical Co. (St. Louis, MO, USA). FOXO1, p-FOXO1, Ac-FOX01, ub-FOX01, LC3, p62, and GAPDH antibodies were purchased from Cell Signaling Technology, Inc. (Beverly, MA, USA). All other chemicals were of analytical grade.

\section{Cell Culture}

The cells were maintained in Dulbecco's modified Eagle's medium (DMEM) supplemented with 10\% fetal calf serum at $37^{\circ} \mathrm{C}$ in an atmosphere containing $5 \% \mathrm{CO} 2$. When cells reached $80 \%-90 \%$ confluence, the cells were added into the DMEM (10\% FCS in media) with different stimulation as indicated.

\section{MTT asay}

The methylthiazoletetrazolium (MTT) reduction assay was used as a qualitative index of cell viability. The effect of resveratrol on cell viability was assessed as percent cell viability compared to resveratrol nontreated control cells, which were arbitrarily assigned $100 \%$ viability.

\section{Assessment of cell apoptosis}

The cytosolic DNA-histone complexes generated during apoptotic DNA fragmentation in the treated QBC939 cells were evaluated using a cell death detection enzyme-linked immunosorbent assay kit (Cell Death Detection ELISA PLUS; Roche Applied Science, Indianapolis, IN, USA), according to the manufacturer's protocol.

\section{ROS}

According to our previous study, we used 2'7'-dichlorofluorescin diacetate (DCFDA) to detect renal ROS production [19]. We incubated each $3-\mu \mathrm{m}$ tissue cryosection with $10 \mu \mathrm{M}$ DCFDA in the dark at $37^{\circ} \mathrm{C}$ for $30 \mathrm{~min}$, before imaging using fluorescence microscopy

\section{KARGER}




\section{Cellular Physiology Cell Physiol Biochem 2018:45:1506-1514 \begin{tabular}{ll|l} 
and Biochemistry Publisned onlIne: February 212018 & $\begin{array}{l}\text { (c) } 2018 \text { The Author(s). Published by S. Karger AG, Basel } \\
\text { www.karger.com/cpb }\end{array}$
\end{tabular}}

\section{Mitochondrial membrane potential}

The MMP of QBC939 was monitored using JC-1, a MMP-sensitive fluorescent dye, as described previously [19]. Briefly, the dissociated QBC939 were washed twice with Hank's balanced salt solution (Sigma, St Louis, MO, USA), and incubated in the dark with JC-1 (7.5 mmol/l; $30 \mathrm{~min}$ at $37^{\circ} \mathrm{C}$, Sigma, St Louis, MO, USA), then cells were washed with JC-1 washing buffer, and fluorescence was detected by fluorescenceassisted cell sorting for QBC939 cells. The relative MMP was calculated using the ratio of J-aggregate/ monomer (590/520 nm).

\section{ATP content measurement}

ATP levels were determined in the QBC939 cells with a luciferase-based bioluminescence assay kit (Sigma-Aldrich, St Louis, MO, USA) in a FLUO star Optima reader according to the manufacturer's instructions. Total ATP levels were the results of the luminescences normalized by protein concentrations.

\section{Western Blotting}

Total cellular proteins from cells of respective group were extracted by lysing cells with buffer containing $150 \mathrm{mM} \mathrm{NaCl}, 0.1 \%$ Triton X-100, 0.5\% Deoxycholate, $0.1 \%$ sodium dodecyl sulfate (SDS), $50 \mathrm{mM}$ Tris-HCl ( $\mathrm{pH} \mathrm{7.0),} \mathrm{and} \mathrm{1mM} \mathrm{ethylenediaminetetraacetic} \mathrm{acid} \mathrm{(EDTA).} \mathrm{Detection} \mathrm{of} \mathrm{protein} \mathrm{expression} \mathrm{by}$ Western blot was carried out according to established protocols. The primary antibodies used were as follows: LC3 (1:1000), p62 (1:2000), and GAPDH (1:1000). The relative intensity of each band, respectively, was normalized to GAPDH.

\section{Statistical analysis}

Quantitative data are presented as mean \pm SD. Statistical significance was determined using one-way analysis of variance (ANOVA); $p<0.05$ was considered statistically significant.

\section{Results}

Endogenous FOXO1 is required for basal and SS-induced autophagy in human cholangiocarcinoma QBC939 cells

To determine whether FOX01 is involved in the autophagic process, we first compared the levels of FOXO1 expression and those of two biological markers of autophagy, namely LC3-II accumulation and p62 degradation under normal conditions and serum starvation (SS) in human cholangiocarcinoma QBC939 cells. Compared to the normal group, FOXO1 levels in SS group cells was elevated and seemed to be related to the autophagic process, as evidenced by increased p62 degradation and LC3-II accumulation (Fig. 1A through C). The autophagy-associated p62 degradation was blocked and LC3-II was further ac-

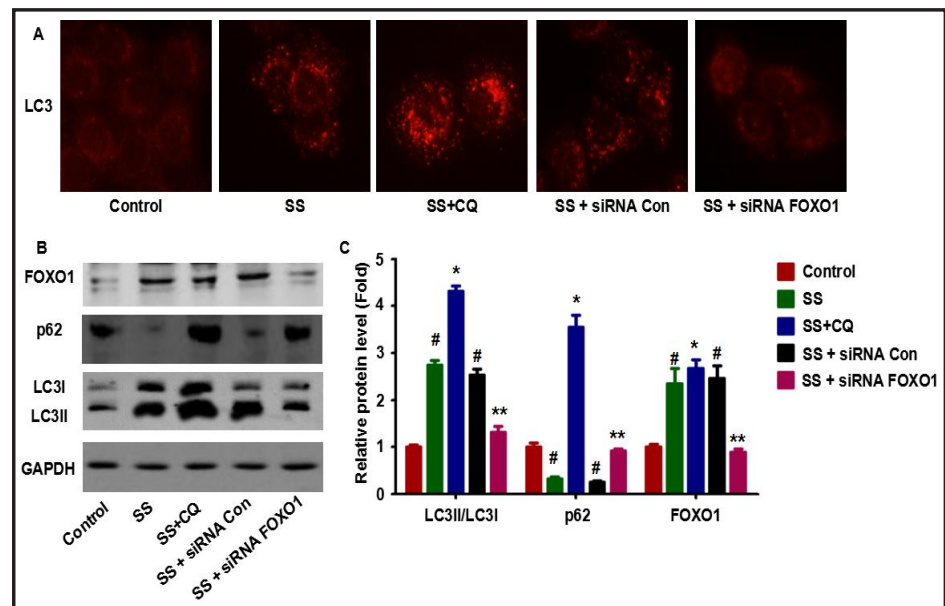

Fig. 1. Knocking down FOXO1 impaired basal and SS-induced autophagy in human cholangiocarcinoma QBC939 cells. (A) Immunofluorescence staining for LC3 in QBC939 cells after various treatments, as indicated. (B) Western blot analysis revealed the expression of LC3II/LC3I, p62, FOX01, and GAPDH proteins in QBC939 cells after various treatments, as indicated. (C) Graphical presentation indicates the relative abundance levels of LC3II/LC3I, p62, and FOX01 after normalization with GAPDH. ${ }^{\#} \mathrm{P}<0.05$ vs. normal control, ${ }^{*} \mathrm{P}<0.05$ vs. SS or $\mathrm{SS}+$ siRNA Con. 
cumulated in the presence of CQ (lysosomal protease inhibitors) in response to SS stimuli (Fig. 1A through C).

To further clarify the role of endogenous FOXO1 in inducing autophagy, a FOXO1 RNA interference (RNAi) or a non-specific control RNAi was transfected into human cholangiocarcinoma QBC939 cells. SS treatment induced p62 degradation and LC3-II accumulation in non-specific RNAi-treated human cholangiocarcinoma QBC939 cells but not in the FOX01 knockdown QBC939 cells (Fig. 1A through C).

Acetylation of FOXO1 and its interaction with Atg 7 is also associated with serum starvation induced autophagy in QBC939 cells

Because a posttranslational modification of cytosolic FOXO1 were reported to be involved in triggering autophagy [7], cytosolic proteins of QBC939 cells were immunoprecipitated with

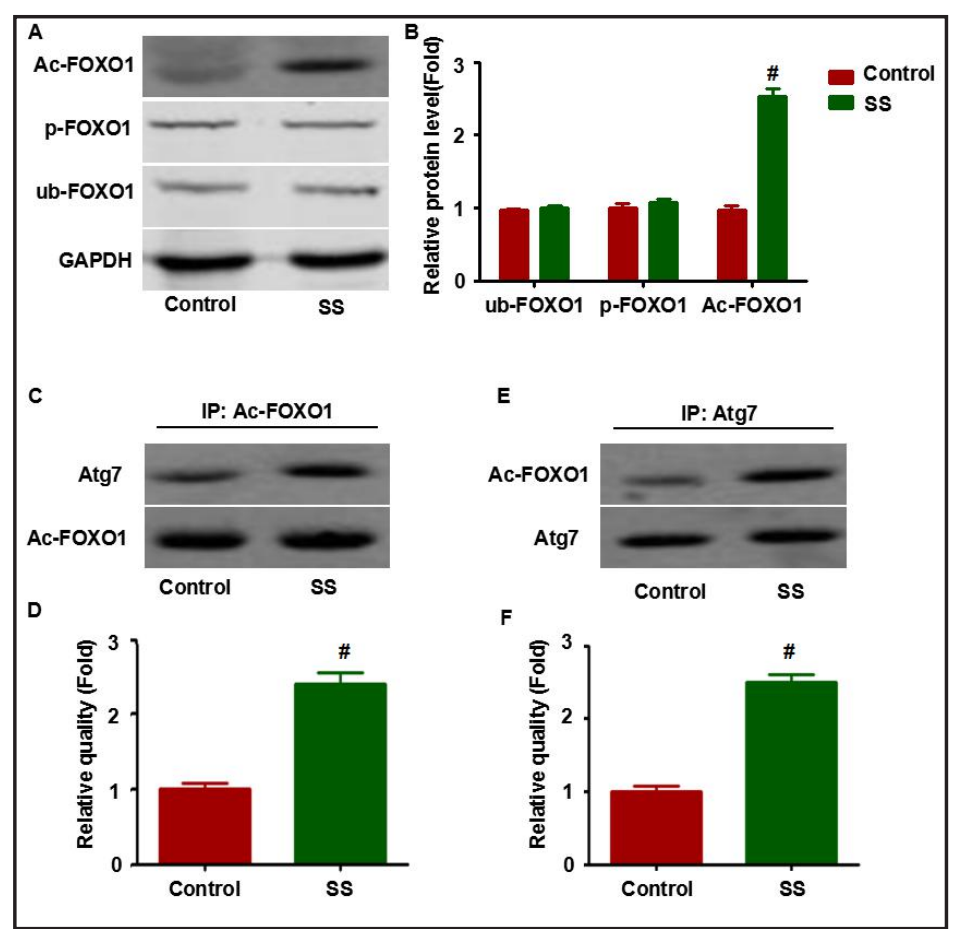

Fig. 2. Acetylation of FOXO1 is also responsible for SS-induced autophagy in QBC939 cells. (A) Western blot analysis revealed the expression of Ac-FOX01, p-FOX01, ub-FOX01, and GAPDH proteins in QBC939 cells under control or SS condition. (B) Graphical presentation indicates the relative abundance levels of Ac-FOX01, p-FOX01, and ub-FOX01 after normalization with GAPDH. (C, E) Co-immunoprecipitation of Atg and Ac-FOX01 in QBC939 cells. Cells were treated as indicated, and the cell lysate was then extracted for co-immunoprecipitation with antiAc-F0X01or Atg7 followed by probing with anti-acetylated Atg7 or AcFOX01 (n=3). (D, F) Graphical presentation shows the relative quality of Atg7 and Ac-FOX01 after normalization with Ac-FOX01 or Atg7 respectively. ${ }^{\text {P }}<0.05$ vs. normal control.

phosphorylation or anti-acetylation antibodies. No detectable changes in ubiquitylation or phosphorylation of cytosolic F0X01 were observed in QBC939 cells before or after the treatments (Fig. 2A and 2B). By contrast, a significant increase in acetylated FOXO1 was detected in serum-starved QBC939 cells (Fig. 2A and 2B). Because a previous report that have proven that FOXO1 acetylation and its interaction with Atg7 is involved in regulating the autophagic process. Subsequently, we examined whether acetylated FOXO1 interacts with Atg7 is associated with enhanced autophay under SS condition. Expectedly, SS enhanced the interaction between endogenous Atg7 and Ac-FOX01 (Fig. 2C through 2F).

Inhibition of acetylation of FOXO1 by Resveratrol imparied autophagic flux in QBC939 cells

Resveratrol is a sirt1 agonist and sirt1 has been confirmed to be responsible for the deacetylation of FOX01[20]. Hence, we explored the possibility that resveratrol could interrupt the autophay process in QBC939 cells. Expectedly, after incubation with $20 \mu \mathrm{M}$ of resveratrol for $48 \mathrm{~h}$, FOXO1 acetylation (Fig. 3A and 3B) and its interaction with Atg7 were attenuated (Fig. 3D and 3E), accompanied by impaired autophagic flux as evidenced by increased LC3 and p62 accumulation (Fig. 3A through 3C). Similar results could be found under SS condition (Data not shown). 
Fig. 3. Resveratrol imparied autophagic flux in QBC939 cells . (A) Western blot analysis revealed the expression of LC3II/LC3I, p62, Ac-FOX01, and GAPDH proteins in QBC939 cells in the presence or absence of Res for 48h. (B) Graphical presentation indicates the relative abundance levels of LC3II/LC3I, p62, and Ac-FOXO1 after normalization with GAPDH. (C) Immunofluorescence staining for LC3 in QBC939 cells after various treatments, as indicated. (D) Co-immunoprecipitation of Atg7 and Ac-FOX01 in QBC939 cells. Cells were treated as indicated, and the cell lysate was then extracted for co-immunoprecipitation with anti-Atg7 followed by probing with anti-Ac-FOXO1 (n=3). (E) Graphical presentation shows the relative quality of Ac-FOXO1 after normalization with Atg7

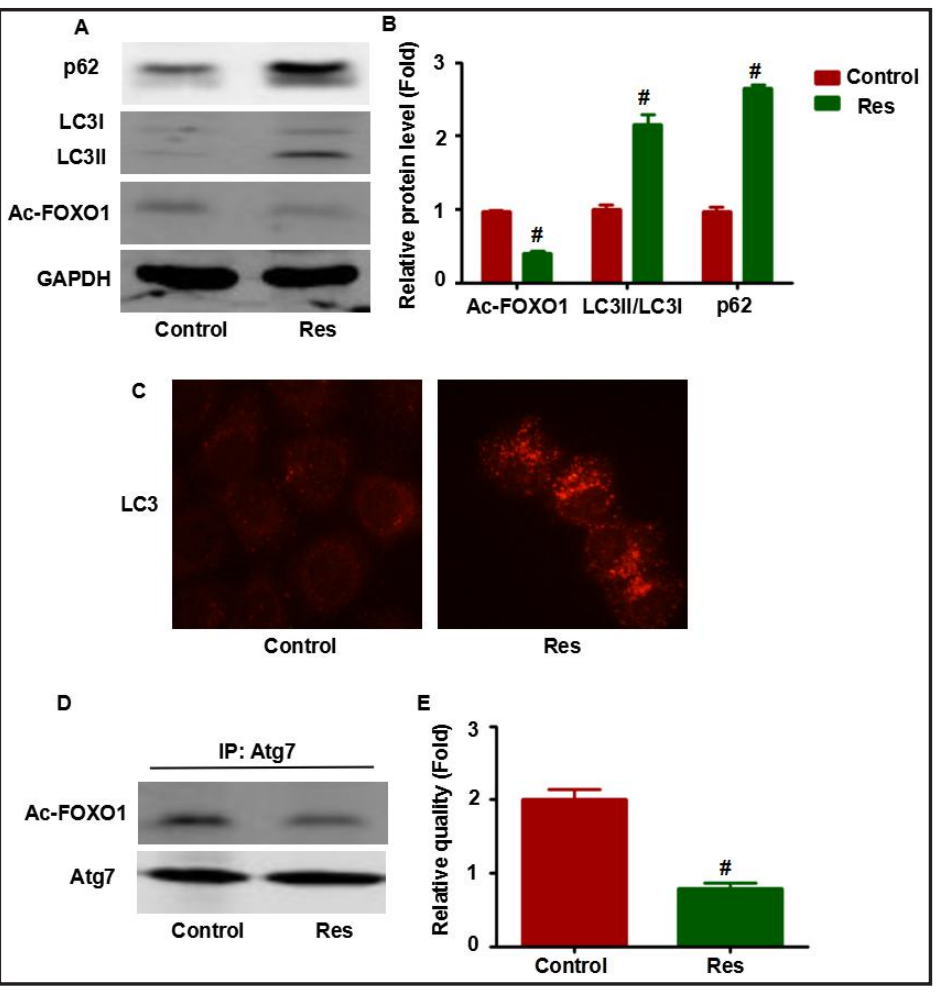
respectively. ${ }^{\#} \mathrm{P}<0.05$ vs. normal control.

Imparied autophagy either by FOXO1 siRNA or resveratrol treatment contributes to decreased cell ability and increased cell apoptosis in QBC939 cells

To further characterize the role of autophagic activity in resveratrol-induced QBC939 cells injury, we also blocked autophagic activity and then assessed apoptosis. Our results showed that addition of $20 \mathrm{uM}$ resverratrol for $48 \mathrm{~h}$ inhibited human cholangiocarcinoma cell viability and increase in cell apoptosis compared with the normal group. Treating QBC939 cells with the autophagy inhibitors 3-Methyladenine (3-MA) resulted in an enhancement of QBC939 damage induced by resveratrol as indicated by the increased apoptosis and decreased cell ability. On the contrary, treatment with Rap, the most frequent strategy used to increase autophagic activity, reduced QBC939 cells damage induced by resverratrol. F0X01 siRNA treatment also decreased cell viability and increased apoptosis in QBC939 cells compared with the group (Fig. 4A through 4D).

Impared autophagy caused either by FOXO1 siRNA or resveratrol treatmentleads to oxidative stress and MtD in QBC939 cells

Since autophagy inhibition could result in oxidative stress [21] and mitochondrial dysfunction [22], both of which have been approved to participate in cell death. We firstly evaluate the ROS production in QBC939 cells after the RES stimuli. Expectedly, ROS level was significantly increased compared with the normal QBC939 cells (Fig. 5A). To evaluate $\mathrm{MtD}$, we used two independent parameters: mitochondrial membrane potential (MMP) and adenosine-50-triphosphate (ATP) levels. MMP collapse has been shown to play an important role in mediation of apoptosis, in which it allows the release of cytochrome c, activation of caspase-9, and subsequently lead to the apoptosis of cells [23]. Second, mitochondria are the energy powerhouses of cells and make the most use of the cell's ATP, tumor cells consumes a large amount of energy supplied as ATP, so any disruption in this supply is likely to cause cell death [15]. Expectedly, resveratrol reduced MMP as indicated by the reduction of JC-1 fluorescence at 590/520 nm (Fig. 5B). Similarly, resveratrol reduced ATP production (Fig. 
5C). Interestedly, RP treatment attenuated the ROS levels and improved the MtD in QBC939 cells, whereas CQ further increased the level of ROS and impaired the MtD in QBC939 cells. FOX01 siRNA treatment also decreased cell viability and increased apoptosis in QBC939 cells compared with the group (Fig. 5A trough 5C).

\section{Discussion}

Cholangiocarcinoma remains one of the most difficult tumors to treat in clinical practice and new therapeutic modalities are currently needed. The induction of cholangiocarcinoma apoptosis is considered to be one promising therapeutic strategy.

It has been recognized that control of autophagy progression in cancer cells is an effective strategy to halt tumor growth [24, 25], as the molecular analyses of human cancers have revealed that autophagy regulators are frequently deregulated in most of the common malignancies [26, 27]. For this reason, autophagy inhibition has been regarded as a promising anticancer strategy to inhibit the multiple cellular processes. Impaired autophagy could influencing the energy metabolism, protein balance, oxidatice stress, and MtD [17, 28, 29]. Hence, knowing the molecular mechanism by which the autophagy is regulated could help us to find a potential therapeutic target to solve this urgent problem.

Regulation of the expression and transcriptional activity of FOXO1 is involved in promoting cellular autophagy [7, 30]. In our study, SS condition could upregulate the expression of FOXO1,which regulate both basal and SS-induced autophagy in QBC939 cells. However agents could directly influencing the expression of FOXO1 is far from clear. Posttranslational modifications including phosphorylation and acetylation of FOXO1 were reported to participate in the regulation of autophagy [31, 32], According to our findings, acetylation of the FOXO1 level and interaction between Ac-FOXO1 and Atg7, which has also been confirmed to promote autophagy, were also found in QBC939 cells and enhanced by SS stimulation. Hence, we speculate that blocking the acetylation of the FOX01 may be a new strategy to inhibit the autophagy process in QBC939 cells.

Resveratrol, a SIRT1 agonist, has recently been acknowledged to activate a mitochondrial apoptotic pathway in human cervical cancer cell $[33,34]$. SIRT1 is a NAd+ dependent deacetylation enzyme. Frampton $\mathrm{G}$ et al. have demonstrated that treatment with progranulin, a secreted growth factor, decreased Sirt1 expression and increased the acetylation of FOX01, resulting in the cytoplasmic accumulation of FOXO1 and enhanced proliferation in

Fig. 4. Inhibiting autophagic flux by targeting FOXO1 inhibits cell viability and induces cell death in QBC939 cells. (A) Western blot analysis revealed the expression of p62 and GAPDH proteins in QBC939 cells after various treatments, as indicated. (B) Graphical presentation indicates the relative abundance levels of p62 after normalization with GAPDH. (C) Cell viability and (D) Apoptotic of QBC939 cells after various treatments, as indicated. Values are presented as the mean \pm standard deviation from six independent experiments. ${ }^{\#} \mathrm{P}<0.05$, vs. Control, ${ }^{*} \mathrm{P}<0.05$ vs. Res or Res+siRNA Con.

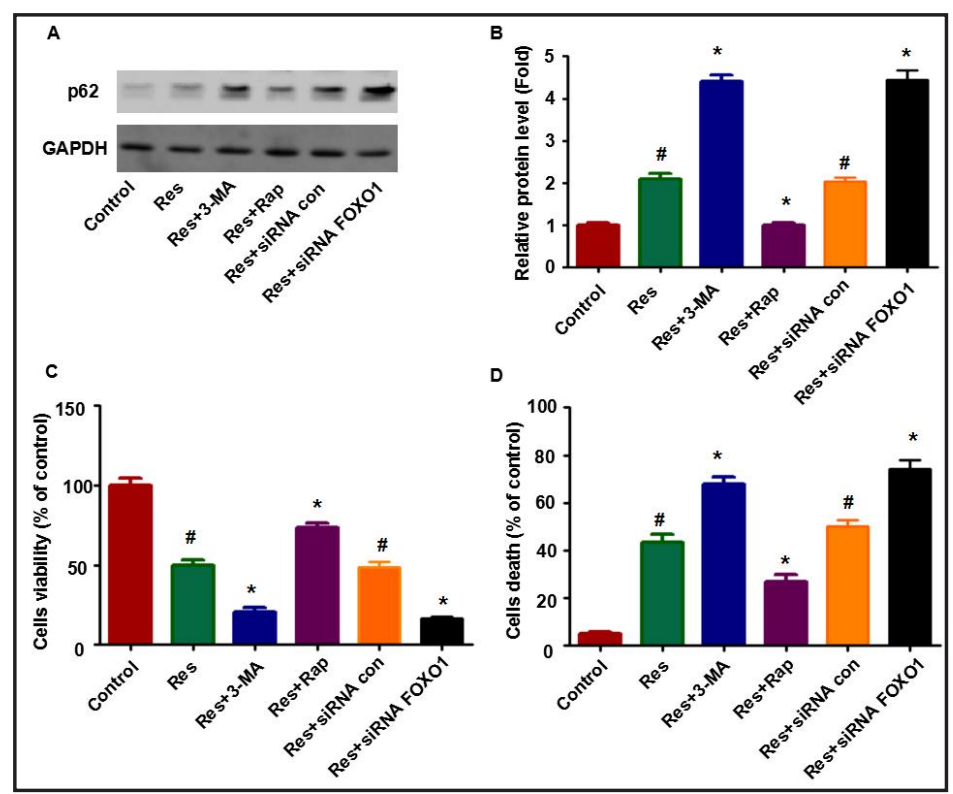


Fig. 5. Inhibiting autophagic flux by targeting FOXO1 worsens oxidative stress and MtD in QBC939 cells . (A) Quantification of 2',7'-dichlorofluorescein (DCF) fluorescence in QBC939 cells after various treatments, as indicated (B) Mitochondrial membrane potential and (C) ATP content were determined. Values are presented the mean \pm standard deviation $(n=6) .{ }^{*} \mathrm{P}<0.05$, vs. Control, ${ }^{*} \mathrm{P}<0.05$ vs. Res or Res+siRNA Con.

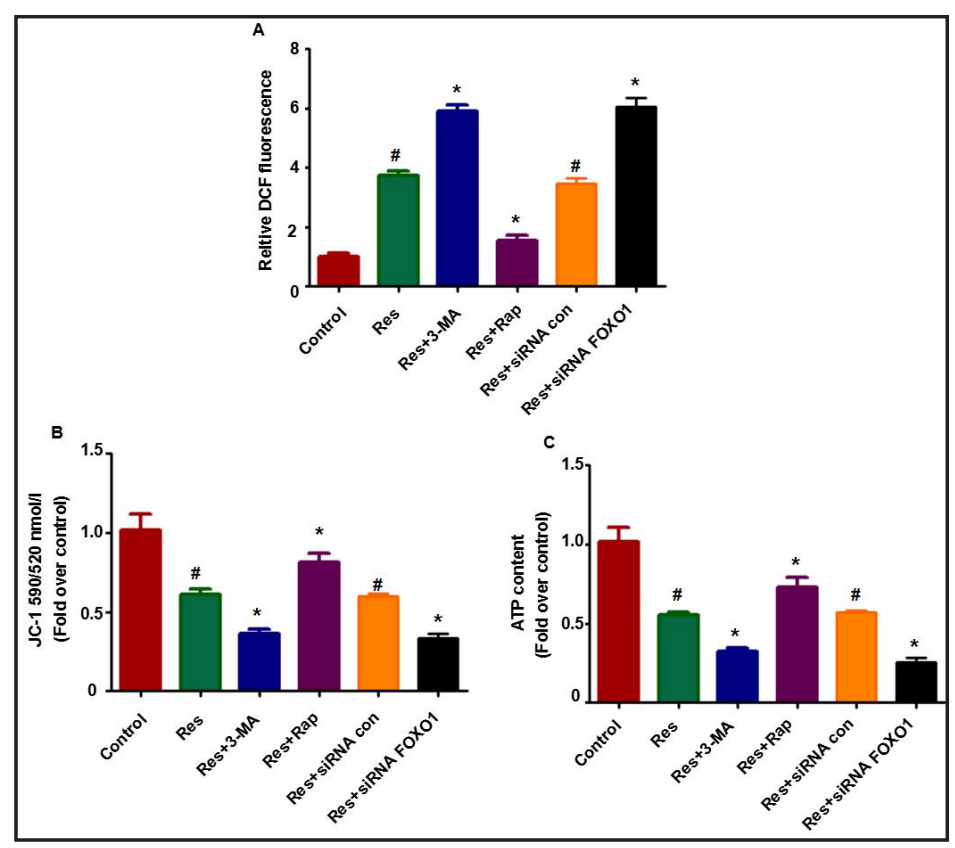

cholangiocytes [35]. However the effect of resveratrol on cholangiocarcinoma is far from clear. In our present investigation, we showed that resveratrol significantly inhibited the acetylation of FOXO1 and interaction between Ac-FOXO1 and Atg7, leading to a blocking autophagy in QBC939 cells, subsequently inducing apoptosis and lower viability of QBC939 cells (Fig. 1 through 3). These results suggested that resveratrol may be examined as an effective chemotherapeutic agent against cholangiocarcinoma.

ROS are known to function as second messengers for signal transduction in many cells and excessive production of ROS can lead to oxidative stress, loss of cell function and apoptosis or necrosis [36, 37]. MtD represents a malfunction in biochemical processes, characterized by MMP collapse, which has been shown to have an essential role in mediation of apoptosis [38]. Furthermore, mitochondria are the energy powerhouses of cells and make the most use of the cell's ATP. Cancer cells consumed a large amount of energy supplied as ATP, so any disruption in this supply is likely to cause apoptosis of cell [39]. Our in vitro data demonstrate that treatment of QBC939 cells with resveratrol inhibited autophagy progression and increased the oxidative stress and MtD (Fig. 2). Additionally, pharmacological inhibition of autophagy worsens resveratrol -induced oxidative stress, MtD, and apoptosis whereas promotion autophagy with RP attenuates these effects, indicating that oxidative stress and MtD results from inhibition of autophagy is one of the mechanisms by which resveratrol induces the apoptosis of QBC939 cells (Fig. 4 and 5).

\section{Conclusion}

Our study provides first evidence thatFOXO1 exists in autophagy regulation and impairing autophagic flux could lead to oxidative stress and MtD in human cholangiocarcinoma QBC939 cells, which indicates that FOXO1 could served as a potential therapeutic target to cure against human cholangiocarcinoma.

\section{Acknowledgements}

This study was supported by the National Natural Science Foundation of China (81172266), the Life Health Technology Foundation of Jiangsu province (BL2012031), and the "333 engineering" Foundation of Jiangsu province (BRA2015472). 


\section{Cellular Physiology Cell Physiol Biochem 2018;45:1506-1514 \begin{tabular}{ll|l} 
and Biochemistry Publisned onlIne: February 212018 & $\begin{array}{l}\text { (c) } 2018 \text { The Author(s). Published by S. Karger AG, Basel } \\
\text { www.karger.com/cpb }\end{array}$ \\
\hline
\end{tabular}}

He et al.: FOXO1 Regulates Autophagic Flux in QBC939 Cells

\section{Disclosure Statement}

The author(s) declare that they have no competing interests

\section{References}

1 Shaib YH, Davila JA, McGlynn K, El-Serag HB: Rising incidence of intrahepatic cholangiocarcinoma in the United States: a true increase? J Hepatology 2004;40:472-477.

2 Landis SH, Murray T, Bolden S, Wingo PA: Cancer statistics, 1998. CA Cancer J Clin1998;48:6-29.

-3 Acton BM, Jurisicova A, Jurisica I, Casper RF: Alterations in mitochondrial membrane potential during preimplantation stages of mouse and human embryo development. Mol Hum Reprod 2004;10:23-32.

-4 Pattingre S, Tassa A, Qu X, Garuti R, Liang XH, Mizushima N, Packer M, Schneider MD, Levine B: Bcl-2 antiapoptotic proteins inhibit Beclin 1-dependent autophagy. Cell 2005;122:927-939.

5 Mizushima N: Autophagy: process and function. Genes Dev 2007; 21:2861-2873.

6 Rubinsztein DC: The roles of intracellular protein-degradation pathways in neurodegeneration. Nature 2006;443:780-786.

7 Wang B, Ding W, Zhang M, Li H, Guo H, Lin L, Chen J, Gu Y: Role of FOXO1 in aldosterone-induced autophagy: a compensatory protective mechanism related to podocyte injury. Oncotarget 2016;7(29):45331-45351.

8 Jash S, Puri V: FoxO1-autophagy axis regulates lipid droplet growth via FSP27. Cell cycle 2016;15:28562857.

-9 Chi HC, Chen SL, Cheng YH, Lin TK, Tsai CY, Tsai MM, Lin YH, Huang YH, Lin KH: Chemotherapy resistance and metastasis-promoting effects of thyroid hormone in hepatocarcinoma cells are mediated by suppression of Fox01 and Bim pathway. Cell Death Dis 2016;7:e2324.

10 Li CF, Zhang WG, Liu M, Qiu LW, Chen XF, Lv L, Mei ZC: Aquaporin 9 inhibits hepatocellular carcinoma through up-regulating FOX01 expression. Oncotarget 2016;7:44161-44170.

$>11$ Wang S, Xia P, Huang G, Zhu P, Liu J, Ye B, Du Y, Fan Z: FoxO1-mediated autophagy is required for NK cell development and innate immunity. Nat Commun 2016;7:11023.

$>12$ Nah J, Yoo SM, Jung S, Jeong EI, Park M, Kaang BK, Jung YK: Phosphorylated CAV1 activates autophagy through an interaction with BECN1 under oxidative stress. Cell Death Dis 2017; 8(5):e2822.

13 Wangpaichitr M, Wu C, Li YY, Nguyen DJM, Kandemir H, Shah S, Chen S, Feun LG, Prince JS, Kuo MT, Savaraj N: Exploiting ROS and metabolic differences to kill cisplatin resistant lung cancer. Oncotarget 2017;8:49275-49292.

14 de Paula Aguiar D, Brunetto Moreira Moscardini M, Rezende Morais E, Graciano de Paula R, Ferreira PM, Afonso A, Belo S, Tomie Ouchida A, Curti C, Cunha WR, Rodrigues V, Magalhães LG: Curcumin Generates Oxidative Stress and Induces Apoptosis in Adult Schistosoma mansoni Worms. PloS one 2016;11:e0167135.

15 Zhang M, Pan H, Xu Y, Wang X, Qiu Z, Jiang L: Allicin Decreases Lipopolysaccharide-Induced Oxidative Stress and Inflammation in Human Umbilical Vein Endothelial Cells through Suppression of Mitochondrial Dysfunction and Activation of Nrf2. Cell Physiol Biochem 2017;41:2255-2267.

-16 Rao W, Zhang L, Peng C, Hui H, Wang K, Su N, Wang L, Dai SH, Yang YF, Chen T, Luo P, Fei Z: Downregulation of STIM2 improves neuronal survival after traumatic brain injury by alleviating calcium overload and mitochondrial dysfunction. Biochim Biophys Acta 2015; 1852:2402-2413.

17 Hashem SI, Murphy AN, Divakaruni AS, Klos ML, Nelson BC, Gault EC, Rowland TJ, Perry CN, Gu Y, Dalton ND, Bradford WH, Devaney EJ, Peterson KL, Jones KL, Taylor MRG, Chen J, Chi NC, Adler ED: Impaired mitophagy facilitates mitochondrial damage in Danon disease. J Mol Cell Cardiol 2017;108:86-94.

18 Golestaneh N, Chu Y, Xiao YY, Stoleru GL, Theos AC: Dysfunctional autophagy in RPE, a contributing factor in age-related macular degeneration. Cell Death Dis 2017;8:e2537.

19 Zhang A, He W, Shi H, Huang X, Ji G: Natural compound oblongifolin C inhibits autophagic flux, and induces apoptosis and mitochondrial dysfunction in human cholangiocarcinoma QBC939 cells. Mol Med Rep 2016;14:3179-3183.

-20 Wang W, Yan C, Zhang J, Lin R, Lin Q, Yang L, Ren F, Zhang J, Ji M, Li Y: SIRT1 inhibits TNF-alpha-induced apoptosis of vascular adventitial fibroblasts partly through the deacetylation of FoxO1. Apoptosis 2013;18:689-701. 


\section{Cellular Physiology Cell Physiol Biochem 2018;45:1506-1514 \begin{tabular}{ll|l} 
DOI: 10.1159/000487576 & $\begin{array}{l}\text { O } 2018 \text { The Author(s). Published by S. Karger AG, Basel } \\
\text { www.karger.com/cpb }\end{array}$ \\
\hline
\end{tabular}}

-21 Li L, Chen J, Sun S, Zhao J, Dong X, Wang J: Effects of Estradiol on Autophagy and Nrf-2/ARE Signals after Cerebral Ischemia. Cell Physiol Biochem 2017;41:2027-2036.

-22 Yao T, Ying X, Zhao Y, Yuan A, He Q, Tong H, Ding S, Liu J, Peng X, Gao E, Pu J, He B: Vitamin D receptor activation protects against myocardial reperfusion injury through inhibition of apoptosis and modulation of autophagy. Antioxid Redox Signal 2015;22:633-650.

23 Lee HH, Park C, Jeong JW, Kim MJ, Seo MJ, Kang BW, Park JU, Kim GY, Choi BT, Choi YH, Jeong YK: Apoptosis induction of human prostate carcinoma cells by cordycepin through reactive oxygen speciesmediated mitochondrial death pathway. Int J Oncol 2013;42:1036-1044.

-24 Sung B, Chung HY, Kim ND: Role of Apigenin in Cancer Prevention via the Induction of Apoptosis and Autophagy. J Cancer Prev 2016;21:216-226.

25 Wang P, Zhu L, Sun D, Gan F, Gao S, Yin Y, Chen L: Natural products as modulator of autophagy with potential clinical prospects. Apoptosis 2017;22:325-356.

26 Leng ZG, Lin SJ, Wu ZR, Guo YH, Cai L, Shang HB, Tang H, Xue YJ, Lou MQ, Zhao W, Le WD, Zhao WG, Zhang X, Wu ZB: Activation of DRD5 (dopamine receptor D5) inhibits tumor growth by autophagic cell death. Autophagy 2017;3:1404-1419.

27 Nakayama S, Karasawa H, Suzuki T, Yabuuchi S, Takagi K, Aizawa T, Onodera Y, Nakamura Y, Watanabe M, Fujishima F, Yoshida H, Morikawa T, Sase T, Naitoh T, Unno M, Sasano H: p62/sequestosome 1 in human colorectal carcinoma as a potent prognostic predictor associated with cell proliferation. Cancer Med 2017;6:1264-1274.

28 Knuppertz L, Osiewacz HD: Autophagy compensates impaired energy metabolism in CLPXP-deficient Podospora anserina strains and extends healthspan. Aging cell 2017;16:704-715.

-29 Goode A, Butler K, Long J, Cavey J, Scott D, Shaw B, Sollenberger J, Gell C, Johansen T, Oldham NJ, Searle MS, Layfield R: Defective recognition of LC3B by mutant SQSTM1/p62 implicates impairment of autophagy as a pathogenic mechanism in ALS-FTLD. Autophagy 2016;12:1094-1104.

-30 Liu L, Zheng LD, Zou P, Brooke J, Smith C, Long YC, Almeida FA, Liu D, Cheng Z: FoxO1 antagonist suppresses autophagy and lipid droplet growth in adipocytes. Cell cycle 2016;15:2033-2041.

-31 Milan G, Romanello V, Pescatore F, Armani A, Paik JH, Frasson L, Seydel A, Zhao J, Abraham R, Goldberg AL , Blaauw B, DePinho RA, Sandri M: Regulation of autophagy and the ubiquitin-proteasome system by the FoxO transcriptional network during muscle atrophy. Nat Commun 2015;6:6670.

32 Vidal RL, Figueroa A, Court FA, Thielen P, Molina C, Wirth C, Caballero B, Kiffin R, Segura-Aguilar J, Cuervo AM, Glimcher LH, Hetz C: Targeting the UPR transcription factor XBP1 protects against Huntington's disease through the regulation of Fox01 and autophagy. Hum Mol Genet 2012;21:2245-2262.

-33 Blanquer-Rossello MD, Hernandez-Lopez R, Roca P, Oliver J, Valle A: Resveratrol induces mitochondrial respiration and apoptosis in SW620 colon cancer cells. Biochim Biophys Acta 2017;1861:431-440.

34 Madreiter-Sokolowski CT, Gottschalk B, Parichatikanond W, Eroglu E, Klec C, Waldeck-Weiermair M, Malli R, Graier WF: Resveratrol Specifically Kills Cancer Cells by a Devastating Increase in the Ca2+ Coupling Between the Greatly Tethered Endoplasmic Reticulum and Mitochondria. Cell Physiol Biochem2016;39:1404-1420.

-35 Frampton G1, Ueno Y, Quinn M, McMillin M, Pae HY, Galindo C, Leyva-Illades D, DeMorrow S: The novel growth factor, progranulin, stimulates mouse cholangiocyte proliferation via sirtuin-1-mediated inactivation of FOXO1.Am J Physiol Gastrointest Liver Physiol 2012;303:G1202-1211.

-36 Moloney JN, Cotter TG: ROS signalling in the biology of cancer. Semin Cell Dev Biol 2017;3:S1084-9521.

-37 Farooqi AA, Li KT, Fayyaz S, Chang YT, Ismail M, Liaw CC, Yuan SS, Tang JY, Chang HW: Anticancer drugs for the modulation of endoplasmic reticulum stress and oxidative stress. Tumour Biol 2015;36:5743-5752.

-38 Sharaf el dein O, Mayola E, Chopineau J, Brenner C: The adenine nucleotide translocase 2, a mitochondrial target for anticancer biotherapy. Curr Drug Targets 2011;12:894-901.

-39 Srinivasan S, Guha M, Kashina A, Avadhani NG: Mitochondrial dysfunction and mitochondrial dynamics-The cancer connection. Biochim Biophys Acta 2017;1858:602-614. 